\title{
Secondary contact zones and hybridizations: the case of the lesser white-toothed shrew (Crocidura suaveolens group, Soricidae)
}

\author{
SYLVAIN DUBEY ${ }^{1 *}$, EBRU DIKER ${ }^{2}$, CENGIZ KURTONUR ${ }^{2}$ and PETER VOGEL ${ }^{3}$ \\ ${ }^{1}$ Heydon-Laurence Building, A08, Science Road, School of Biological Sciences, University of Sydney, \\ Sydney, NSW 2006, Australia \\ ${ }^{2}$ Department of Biology, Faculty of Art and Sciences, Trakya University, Edirne, Turkey \\ ${ }^{3}$ Department of Ecology and Evolution, University of Lausanne, 1015 Lausanne, Switzerland
}

Received 30 November 2007; accepted for publication 19 March 2008

\begin{abstract}
In the present study, we analyzed 58 samples of the lesser white-toothed shrew group (Crocidura suaveolens) from eastern Europe and Turkey, where, according to previous publications, three different mitochondrial and nuclear lineages are present. We sequenced $799 \mathrm{bp}$ of the nuclear BRCA1 gene and $400 \mathrm{bp}$ of the mitochondrial cytochrome $b$ gene to: (1) determine a potential contact zone between the lineages; (2) detect hybridizations and introgressions between them; and (3) comment on the level of reproductive isolation of the different lineages. We revealed two zones of hybridization in Turkey, of which the first occurred west of the Bosphorus Straits (three hybrids) and the second in Anatolia (twelve hybrids). In the latter, the nuclear markers revealed a large zone of hybridization, of approximately $600 \mathrm{~km}$. It also revealed that hybrids of first, second, and later generations are present within the populations, and therefore that the reproductive isolation between the different lineages is weak. (C) 2008 The Linnean Society of London, Biological Journal of the Linnean Society, 2008, 95, 557-565.
\end{abstract}

ADDITIONAL KEYWORDS: BRCA1 - cytochrome $b$ - dispersal - expansion - hybrid - mammals phylogeography.

\section{INTRODUCTION}

Numerous phylogeographic studies have shown the impact of Pleistocene climatic fluctuations throughout the world. In the northern hemisphere, mitochondrial DNA (mtDNA) markers reveal general patterns that involve southern refugia and northern recolonization routes (Hewitt, 2000, 2004a, b). Mountain chains and seas are known to have isolated populations in different glacial refugia, leading to the formation of divergent genetic lineages, which are often considered as different subspecies or species. Moreover, the Pleistocene climatic fluctuations have led to various postglacial recolonization patterns of those lineages (Taberlet et al., 1998; Hewitt, 1999). Western Eurasian

*Corresponding author.

E-mail: sylvain.dubey@bio.usyd.edu.au refugia include the Iberian Peninsula, the ItaloBalkanic region, and eastern areas such as the Caucasus, western Asia, and possibly northern refugia such as the southern Urals and Carpathian Mountains (Santucci, Emerson \& Hewitt, 1998; Taberlet et al., 1998; Nesbo et al., 1999; Palme \& Vendramin, 2002; Seddon et al., 2002; Michaux et al., 2004; Culling et al., 2006; Dubey et al., 2006, 2007a, b; Saarma et al., 2007). In some cases, the subsequent postglacial expansion led to secondary contact zones and genetic introgressions between populations derived from separated glacial refugia; for example, in the bank vole Clethrionomys glareolus (Tegelström \& Jaarola, 1998), in the hares Lepus timidus and L. europaeus (Thulin, Fang \& Averianov, 2006), and in the ground squirrel Spermophilus (Ermakov et al., 2002).

Unfortunately, most phylogeographic studies are based on mtDNA and consider at most the phenotype, 
whereas the inclusion of nuclear markers can considerably enhance our understanding of population history, as shown recently in several species of Palaearctic shrews of the genus Crocidura (Brändli et al., 2005; Bannikova et al., 2006; Dubey et al., 2006, 2007b). Using these techniques, secondary contact zones and mitochondrial introgressions can be more easily detected. In addition, this is the only way to reveal hybridization between different lineages and thus to determine the level of reproductive isolation between taxa.

Within the lesser white-toothed shrew group (Crocidura suaveolens Pallas, 1811) numerous subspecies and species have been described based on morphological characteristics (Corbet, 1978; Hutterer, 2005). However, in studies based on mitochondrial and/or nuclear phylogenetics, Dubey et al. (2006, 2007a) have highlighted only ten well differentiated mitochondrial lineages, originating from various Pleistocene refugia. Nuclear and mitochondrial datasets were congruent for the seven different clades identified in Dubey et al. (2006). Moreover, two pairs of morphologically recognized species, namely (1) Crocidura sibirica Dukelski, 1930 from Siberia and the European C. suaveolens from Crimea (type locality) and (2) Crocidura monacha Thomas, 1906 and Crocidura gueldenstaedtii Pallas, 1811, both from the Near East, were revealed to be genetically identical and should therefore be considered as synonyms from a genetic point of view (Bradley \& Baker, 2001; Baker \& Bradley, 2006).

Nevertheless, the specific status of these lineages remains uncertain, and the mean genetic distance between them (Kimura two-parameters distance $=$ $4.7 \%$ and $10.8 \%$, respectively) may be situated within a single species, or represent an incipient species or a well separated full species (Bradley \& Baker, 2001).

Based on a large sample, the geographic distribution of lineages was well defined, allowing approximate delineation of the potential contact zones. Nevertheless, no sympatry was detected. However, in a study based on nuclear and mitochondrial markers and focused on the Caucasus region, Bannikova et al. (2006) found one hybrid (mitochondrial introgression) between the lineages gueldenstaedtii and suaveolens. Unfortunately, very few samples were analyzed in the potential contact zones, making it difficult to comment on the level of isolation of the different lineages. Nonetheless, the authors drew taxonomic conclusions, proposing levels of classification such as 'superspecies', 'supersubspecies', and 'semispecies'.

In the present study, we analyzed samples of the C. suaveolens group from western Turkey, where three different mitochondrial and nuclear lineages are present (Dubey et al., 2006, 2007a). The aims of the study were: (1) to determine a potential contact zone between the lineages; (2) to detect hybridizations and introgressions between them; and (3) to comment on the level of reproductive isolation of the different lineages.

\section{MATERIAL AND METHODS}

SAMPLING

We analyzed 58 samples of the Crocidura suaveolens group collected in eastern Europe and western Turkey (Fig. 1, Table 1). We used as an outgroup Crocidura brunnea and Crocidura nigripes (cyt-b: DQ630385 and DQ630384, respectively) from Indonesia, two species that are included within the sister clade of the C. suaveolens group (Dubey et al., 2007c). This set of samples included material from the collections of the University of Lausanne, Lausanne, Switzerland (IZEA) and from the collection of the Trakya University, Edirne, Turkey. Some additional sequences were obtained from Dubey et al. (2006; Table 1).

\section{DNA EXTRACTION AND AMPLIFICATION}

Liver samples from the IZEA collection were frozen in liquid nitrogen in the field and kept for several years at $-70{ }^{\circ} \mathrm{C}$ before being stored in ethanol for DNA extraction. Samples from the other collections were stored directly in ethanol. The DNA extraction was carried out using the QIA Amp DNA Mini Kit (Qiagen). Double-stranded DNA amplifications of the cytochrome $b$ gene (cyt-b) were performed using the primer pair L14724/H15149 (Irwin, Kocher \& Wilson, 1991). Amplifications of the breast cancer susceptibility 1 gene (BRCA1) were performed using the primer pair BRCA1f/BRCA1r18 (Dubey et al., 2006). Amplification conditions for BRCA1 and cyt-b consisted of 40 thermal cycles of denaturation at $94{ }^{\circ} \mathrm{C}$ for $60 \mathrm{~s}\left(30 \mathrm{~s}\right.$ for cyt-b), annealing at $50{ }^{\circ} \mathrm{C}$ for $60 \mathrm{~s}\left(45 \mathrm{~s}\right.$ for cyt-b), and extension at $72{ }^{\circ} \mathrm{C}$ for $120 \mathrm{~s}$ $(60 \mathrm{~s}$ for $c y t-b)$. The polymerase chain reaction (PCR) products were checked on a $1 \%$ agarose gel and then purified using the QIAquick PCR Purification Kit (Qiagen) following the manufacturer's instructions. DNA sequencing was performed in a total volume of $10 \mu \mathrm{l}$ containing 1-3 $\mu \mathrm{l}$ of amplified PCR product, $1 \mu \mathrm{l}$ of $10 \mu \mathrm{M}$ primer, and $4 \mu \mathrm{l}$ of $\mathrm{ABI}$ PRISM Dye Terminator 1 (Perkin-Elmer). Sequence reactions were visualized on an ABI 3100 genetic analyzer (Applied Biosystems).

\section{PHYLOGENETIC METHODS}

Nucleotide sequences of $c y t-b$ and BRCA1 genes were edited using Sequence Navigator (Parker, 1997) and 


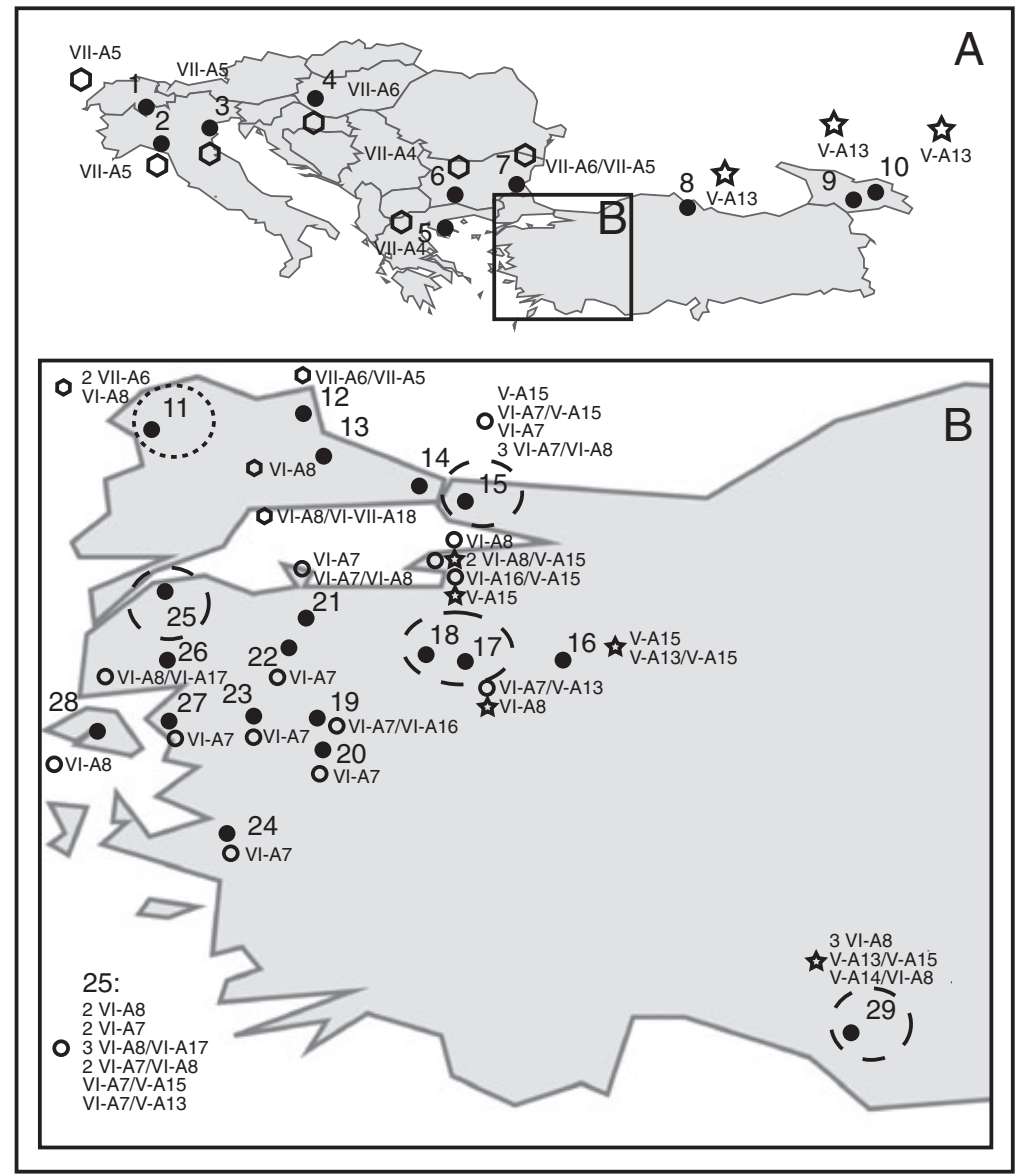

Figure 1. Location of populations (black round) with mitochondrial lineages (star, V; circle, VI; hexagon, VII) and nuclear lineages and alleles of each sample (V-A13, V-A14, V-A15, VI-A7, VI-A8, VI-A17, VI-A16, VII-A4, VII-A5, VII-A6, VI-VII-A18). Populations included within a circle are those possessing haplotypes or alleles of two different lineages (eastern of the Bosphorus Strait, V and VI; western of the Bosphorus Strait, VI and VII).

manually aligned. Two methods of phylogenetic analysis were performed for cyt-b, using PAUP*version 4.0b10 PPC (Swofford, 1998). A Neighbour-joining (NJ) tree was constructed using Kimura twoparameter genetic distances (Kimura, 1980). Parsimony analyses (MP) were performed using the options: heuristic search, stepwise addition of sequences, ten replicates of random additions of taxa, and treebisection-reconnection branch swapping (Swofford, 1998). Tests were conducted on the complete fragment, all codon positions were used, and trees were rooted using sequences from $C$. brunnea and C. nigripes. Fast maximum likelihood (ML) heuristic searches and bootstrap analyses (1000 replicates) were performed using PHYML (Guindon \& Gascuel, 2003) with a general time reversible model, which had been selected previously using MODELTEST 3.06 according to the protocol of Posada \& Crandall (1998). Bootstrap support values were obtained with 1000 pseudoreplicates.

\section{RESULTS}

\section{MitOCHONDRIAL DATA}

In 47 sequences of $400 \mathrm{bp}$ from samples of the $C$. suaveolens group, thirteen haplotypes were found, and were named H1 to H13 (Fig. 2, Table 1). They corresponded to the three different lineages $\mathrm{V}$ (H1H2), VI (H7-H13), and VII (H3-H6) found by Dubey et al. (2006, 2007a). The sequences are deposited under the Genbank accession numbers EU271921EU271933. Lineage V was supported by bootstrap values of $98 \%$ for ML, 99\% for MP, and $100 \%$ for NJ; lineage VI by values of $100 \%$ for ML, $97 \%$ for MP, and $99 \%$ for NJ; lineage VII by values of $81 \%$ for ML, $66 \%$ for MP, and $90 \%$ for NJ.

In the present study, lineage $\mathrm{V}$ was found to be distributed from western Turkey (east of the Bosphorus) to Georgia; lineage VI was found in western Turkey (east of the Bosphorus) and on Lesvos Island (Greece); and lineage VII was distributed from 
$10201004+\sum^{2}$ 乙 $:$ б

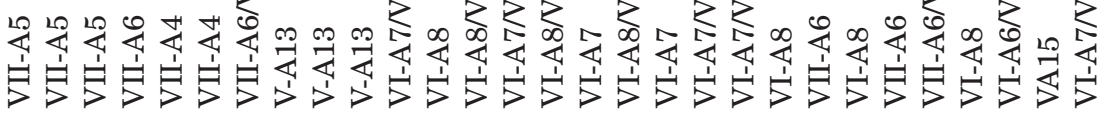

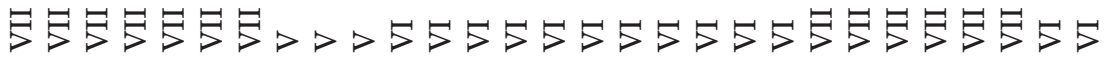

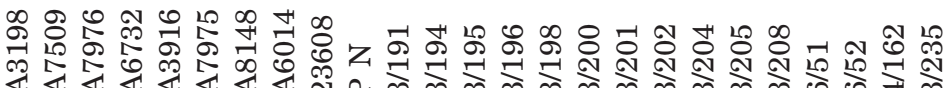

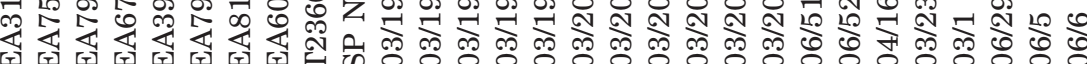

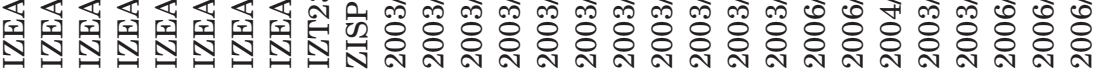

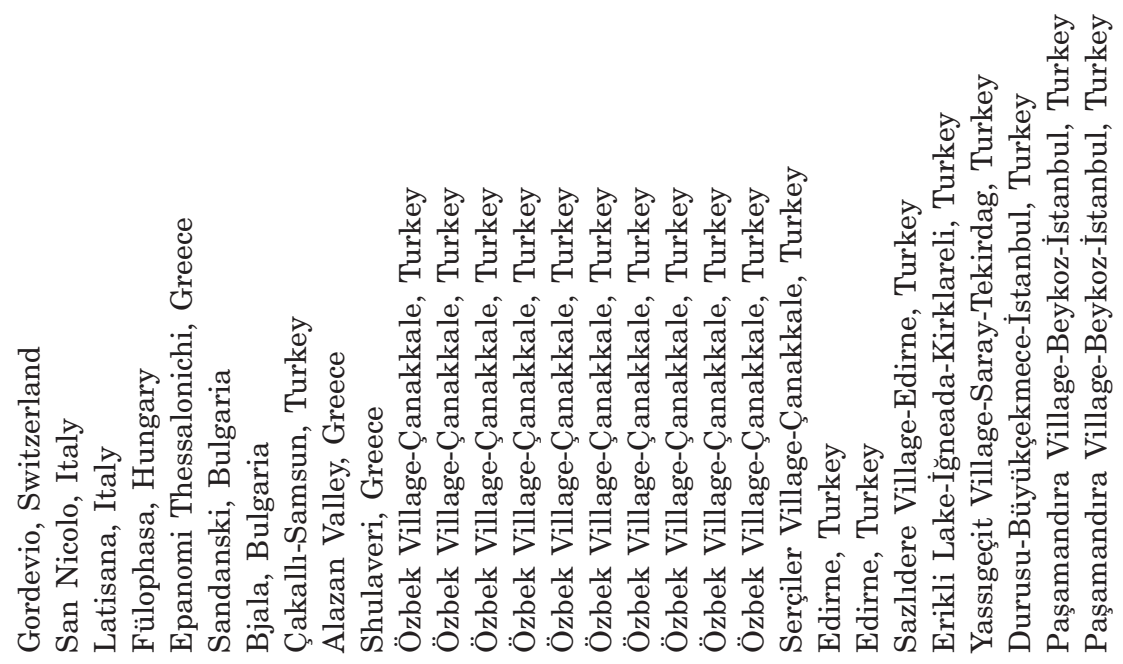




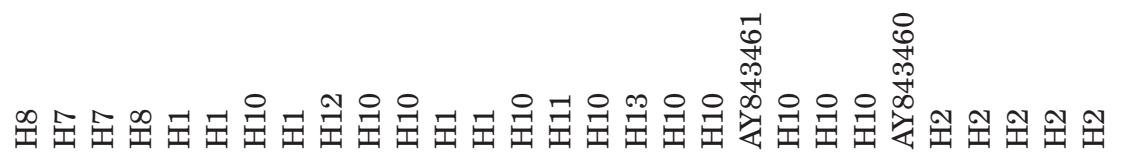

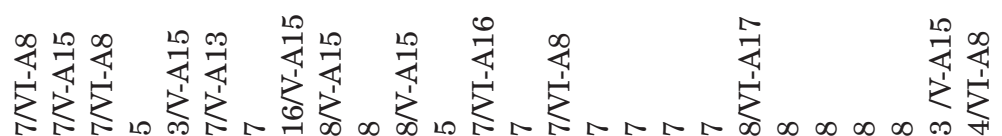

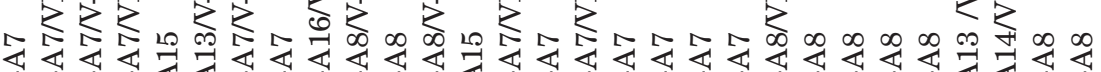

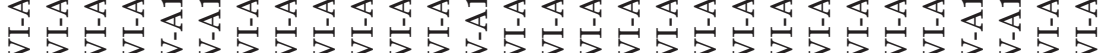

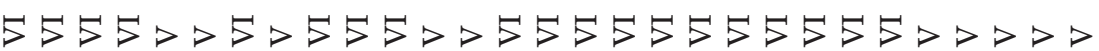

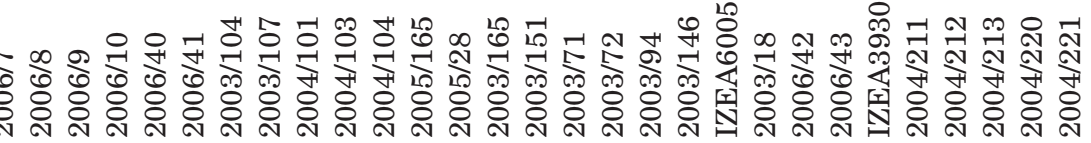

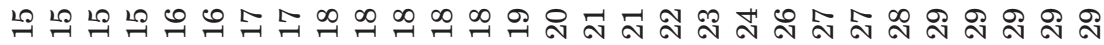

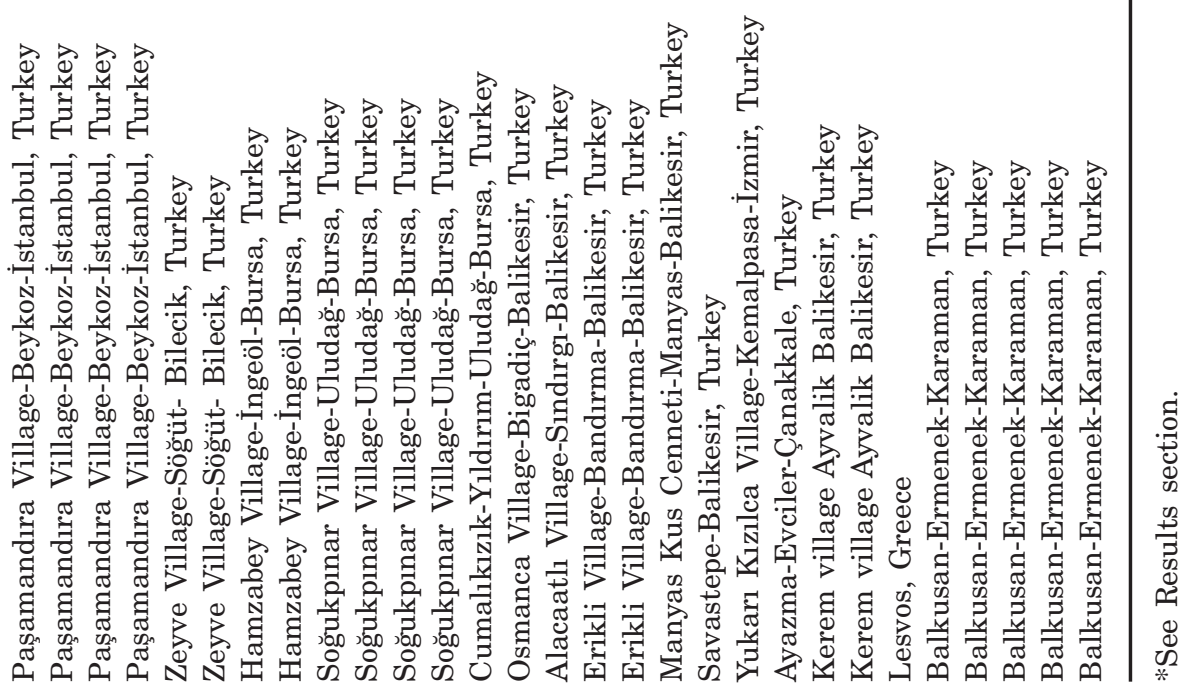




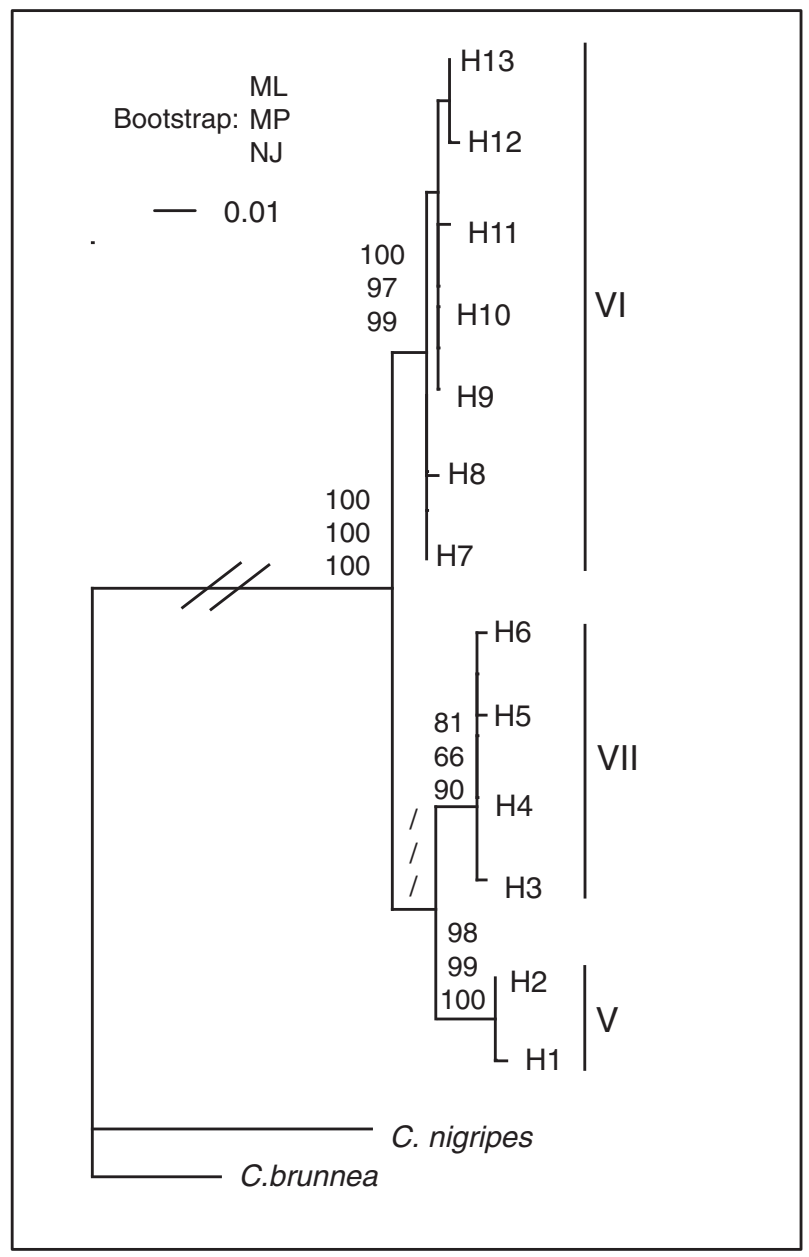

Figure 2. Phylogeny of the 400 bp cytochrome $b$ fragment analysed with maximum likelihood, using the general time reversible model of substitution and tree-bisectionreconnection branch swapping. Values in branches are bootstrap indices of support for the major branches for maximum likelihood (ML), parsimony (MP) and Neighbour-joining (NJ) analyses (percentage of 1000). Codes are as in Table 1.

Switzerland to western Turkey (west of the Bosphorus). All populations showed only haplotypes of one lineage, except in western Turkey, east of the Bosphorus, where haplotypes of lineages V and VI were found within the same locality (Hamzabey and Soğukpınar; Fig. 1, localities 17 and 18, respectively).

Sequences of cyt-b obtained by Dubey et al. (2006) (Table 1) were not included in the phylogeny shown in Figure 2 because the sequences were not entirely overlapping. Nevertheless, they were used to determine the different existing lineages in the present study (results not shown).

\section{NUCLEAR DATA}

From 799 bp sequences of the BRCA1 gene, we found 11 different alleles within the studied areas, which were named A4 to A18. Six alleles corresponded to alleles previously found in Dubey et al. (2006): A4 to A6 of lineage VII, A7 and A8 of lineage VI, and A13 of lineage V. For convenience, the same nomination is used in the present study, and the five newly identified alleles are named A14 to A18. The number of mutations varied from one to six between the different alleles (Table 2); three characteristic mutations separated the alleles of lineage $\mathrm{V}$ from those of lineages VI and VII. Alleles A13 to A15 were found in Turkey and Georgia and were associated with mitochondrial lineage V. Alleles A7, A8, A16, and A17 were found in western Turkey and were associated with mitochondrial lineage VI, and alleles A4 to A6 were found in Europe and were associated with mitochondrial lineage VII. Allele A18 exhibited the characteristic mutations of lineages VI and VII. However, because it was found in only one sample, from the west of the Bosphorus, in association with allele A8 and a haplotype of lineage VII, it was not possible to classify this allele within lineage VI or VII. The eleven alleles are named as follows in Tables 1 and 2 and Figure 1: V-A13, V-A14, V-A15, VI-A7, VI-A8, VI-A17, VI-A16, VII-A4, VII-A5, VII-A6, and VI-VIIA18. The sequences are deposited under Genbank accession numbers EU271910-EU271920.

In Turkey, six different populations showed alleles of two different nuclear lineages, as detailed below.

Population 17 (Fig. 1; Hamzabey): one homozygote VI with the haplotype lineage $\mathrm{V}$, and one heterozygote V/VI with the haplotype lineage VI were found (Fig. 1, Table 1).

Population 18 (Soğukpınar): one homozygote VI with the haplotype lineage VI, one homozygote V with the haplotype lineage $\mathrm{V}$, two heterozygotes V/VI with the haplotype lineage VI, and one heterozygote with the haplotype lineage $\mathrm{V}$ were found.

Population 25 (Özbek): nine samples of homozygotes VI with the haplotype lineage VI, and two heterozygotes V/VI with the haplotype lineage VI were found.

Population 15 (Paşamandıra): four homozygotes VI with the haplotype lineage VI, one homozygote $\mathrm{V}$ with the haplotype lineage VI, and one heterozygote V/VI with the haplotype lineage VI were found.

Population 29 (Balkusan): three homozygotes VI, one homozygote V, and one heterozygote V/VI with the haplotype lineage $\mathrm{V}$ were found. 
Table 2. Mutations observed between the different alleles of BRCA1 and location in bp

\begin{tabular}{|c|c|c|c|c|c|c|c|c|c|c|c|c|c|}
\hline Lineage and allele & 28 & 122 & 154 & 171 & 245 & 526 & 531 & 581 & 589 & 631 & 656 & 735 & 740 \\
\hline V-A13 & A & G & $\mathrm{C}$ & A & $\mathrm{T}$ & G & G & G & $\mathrm{T}$ & G & A & A & G \\
\hline V-A14 & . & . & . & . & . & . & . & . & . & A & . & . & . \\
\hline V-A15 & . & . & . & . & $\mathrm{C}$ & . & . & . & . & . & . & . & . \\
\hline VI-A7 & . & A & . & G & $\mathrm{C}$ & . & $\mathrm{A}$ & . & . & . & . & $\mathbf{T}$ & $\mathbf{A}$ \\
\hline VI-A8 & . & . & . & G & $\mathrm{C}$ & . & . & . & . & . & . & $\mathbf{T}$ & $\mathbf{A}$ \\
\hline VI-A16 & . & . & . & G & $\mathrm{C}$ & . & . & A & . & . & . & $\mathbf{T}$ & $\mathbf{A}$ \\
\hline VI-A17 & . & . & $\mathrm{T}$ & G & $\mathrm{C}$ & . & . & . & . & . & . & $\mathbf{T}$ & $\mathbf{A}$ \\
\hline VII-A4 & . & . & . & G & $\mathrm{C}$ & . & . & . & . & . & $\mathrm{T}$ & $\mathbf{T}$ & $\mathbf{A}$ \\
\hline VII-A5 & $\mathrm{C}$ & . & . & G & $\mathrm{C}$ & . & . & . & . & . & . & $\mathbf{T}$ & $\mathbf{A}$ \\
\hline VII-A6 & $\mathrm{C}$ & . & . & G & $\mathrm{C}$ & A & . & . & . & . & . & $\mathbf{T}$ & $\mathbf{A}$ \\
\hline VI-VII-A18* & . & . & . & G & $\mathrm{C}$ & . & . & . & $\mathrm{C}$ & . & . & $\mathbf{T}$ & $\mathbf{A}$ \\
\hline
\end{tabular}

Characteristic mutations of the lineages VI and VII are shown in bold.

*See Results section.

Population 11 (Edirne): two homozygotes VI with the haplotype lineage VII and one homozygote VI with the haplotype lineage VII were found.

\section{DISCUSSION}

According to Dubey et al. (2006, 2007a), three different mitochondrial and nuclear lineages, designated $\mathrm{V}$ to VII, of the lesser white-toothed shrew are present in eastern Europe and Turkey. The mitochondrial Kimura two-parameter genetic distance between the lineages is $5.8 \%$ for V/VI, $4.7 \%$ for VI/VII, and $5.1 \%$ for V/VII. These lineages are known to have a parapatric distribution: lineage $\mathrm{V}$ is distributed strictly in central and eastern Turkey, lineage VI in western Anatolia, and lineage VII in central and eastern Europe.

Based on thorough sampling in this area, the present study has highlighted more complex relationships between the mitochondrial and nuclear data than those reported previously by Dubey et al. (2006, 2007a). Unexpectedly, we revealed hybridizations between lineages VI and VII in western Turkey, west of the Bosphorus, where three samples from different populations possessed haplotypes of lineage VII and nuclear alleles of lineage VI (Fig. 1). By contrast, lineage VI was only found east of the Bosphorus strait. From a biogeographic point of view, this means that lineage VI, during its postglacial expansion from a refugium situated in Anatolia (Dubey et al., 2006), crossed the Bosphorus strait and colonized eastern Europe. This type of colonization from Turkey to Europe is poorly documented by current phylogeographic studies; it has only been described previously for the bicolored shrew Crocidura leucodon (Dubey et al., 2007b). However, the permeability of the Bosphorus for various species has already been demonstrated for the reverse direction in classical zoogeography (Hosey, 1982).
Similarly, hybridizations between lineages $\mathrm{V}$ and VI were detected in western and southern central Turkey, with 12 hybrids possessing mitochondrial haplotypes and nuclear alleles of one of the respective lineages, and some being heterozygotes or homozygotes (i.e. hybrids of first, second, and/or later generations). The nuclear data revealed a large zone of hybridization; the most distant populations sharing the two lineages were separated by approximately $600 \mathrm{~km}$ (Fig. 1).

Interestingly, the mitochondrial data revealed a much smaller introgression zone: the only two populations sharing the two different haplotype lineages were separated by less than $50 \mathrm{~km}$. Consequently, the mitochondrial introgression is very limited compared with the nuclear one, despite the fact that mtDNA is not linked directly to genes that are involved in reproductive isolation, and may penetrate reproductive barriers more easily than nuclear DNA (Barton \& Jones, 1983; Takahata \& Slatkin, 1984; Tegelström \& Jaarola, 1998).

The present study failed to demonstrate clear reproductive isolation between three different lineages of the $C$. suaveolens group, despite $c y t-b$ distances of $4.7 \%$ to $5.8 \%$ between them, and an origin dating from the Lower Pleistocene (Dubey et al., 2006, 2007a). It is probable that the absence of karyotypic rearrangement between the different lineages of the C. suaveolens group supported these types of introgression between relatively distant sister taxa.

In a recent study employing nuclear and mitochondrial markers, Bannikova et al. (2006) translated the different lineages of $C$. suaveolens s.l. into taxonomic units and attributed levels (superspecies, supersubspecies, semispecies) in accordance with the branching pattern of the resulting phylogeny. By contrast, Dubey et al. $(2006,2007 \mathrm{a})$ preferred to avoid any splitting as long as the degree of genetic isolation at 
the contact zones is unknown. In the present study, the results obtained provide evidence of conspecificity between lineages V to VII. It is probable that lineages IX (Crocidura suaveolens aleksandrisi from Lybia) and X (Crocidura suaveolens cypria from Cyprus Island; Dubey et al., 2007a), which show a similar level of differentiation, should be considered as conspecific, although they are geographically isolated and no contact zones have been identified.

The study of contact zones between deeper branches of the C. suaveolens group, i.e. between lineages II (Crocidura suaveolens suaveolens) and III (Crocidura suaveolens caspica), or of both with lineage V (Crocidura suaveolens gueldenstaedtii), or between lineages IV (Crocidura suaveolens iculisma) and VIII (Crocidura suaveolens mimula), will be a challenge. Nevertheless, no thorough sampling in potential contact zones is currently possible. In conclusion, we have revealed that the clear large-scale biogeographic pattern, shown in Dubey et al. (2006, 2007a), with parapatric distributions of different genetic lineages of lesser white-toothed shrews, is too simplistic. Therefore, sampling in potential contact zones coupled with the analyses of mitochondrial and nuclear markers is the only way to reveal a clear phylogeographic pattern, as well as the level of reproductive isolation between closely-related taxa. Consequently, our results support the argument that both nuclear and mitochondrial markers should be included in phylogenetic studies because the full story can be more complex than the analysis of either category of marker alone might indicate.

\section{ACKNOWLEDGEMENTS}

We thank Nelly Di Marco for laboratory facilities and Darron Cullen for linguistic advice. This work was supported by the Herbette Foundation, University of Lausanne.

\section{REFERENCES}

Baker RJ, Bradley RD. 2006. Speciation in mammals and the genetic species concept. Journal of Mammalogy 87: 643-662.

Bannikova AA, Lebedev VS, Kramerov DA, Zaitsev MV. 2006. Phylogeny and systematics of the Crocidura suaveolens species group: corroboration and controversy between nuclear and mitochondrial DNA markers. Mammalia 70: 106-119.

Barton N, Jones JS. 1983. Mitochondrial-DNA-New clues about evolution. Nature 306: 317-318.

Bradley RD, Baker RJ. 2001. A test of the genetic species concept: cytochrome-b sequences and mammals. Journal of Mammalogy 82: 960-973.

Brändli L, Handley LJ, Vogel P, Perrin N. 2005. Evolu- tionary history of the greater white-toothed shrew (Crocidura russula) inferred from analysis of mtDNA, Y and X chromosome markers. Molecular Phylogenetics and Evolution 37: 832-844.

Corbet GB. 1978. The mammals of the Palaearctic Region: a taxonomic review. London, Ithaca: British Museum (Natural History), Cornell University Press.

Culling MA, Janko K, Boron A, Vasil'ev VP, Cote IM, Hewitt GM. 2006. European colonization by the spined loach (Cobitis taenia) from Ponto-Caspian refugia based on mitochondrial DNA variation. Molecular Ecology 15: 173190.

Dubey S, Cosson J-F, Magnanou E, Vohralík V, Hutterer R, Vogel P. 2007a. Mediterranean populations of the Lesser white-toothed shrew (Crocidura suaveolens group): an unexpected puzzle of Pleistocene survivors and prehistoric introductions. Molecular Ecology 16: 3438-3452.

Dubey S, Cosson J-F, Vohralik V, Krystufek B, Diker E, Vogel P. 2007b. Molecular evidence of Pleistocene bidirectional faunal exchange between Europe and the Near East: the case of the bicolored shrew (Crocidura leucodon, Soricidae). Journal of Evolutionary Biology 20: 1799-1808.

Dubey S, Salamin N, Ohdachi SD, Barrière P, Vogel P. 2007c. Molecular phylogenetics of Soricidae (Mammalia, Eulipotyphla) reveals timing of transcontinental colonisations. Molecular Phylogenetics and Evolution 44: 126-137.

Dubey S, Zaitsev M, Cosson J-F, Abdukadier A, Vogel P. 2006. Pliocene and Pleistocene diversification and multiple refugia in a Eurasian shrew (Crocidura suaveolens group). Molecular Phylogenetics and Evolution 38: 635-647.

Ermakov OA, Surin VL, Titov SV, Tagiev AF, Luk AV. 2002. A molecular genetic study of hybridization in four species of ground squirrels (Spermophilus: Rodentia, Sciuridae). Russian Journal of Genetics 38: 796-809.

Guindon S, Gascuel O. 2003. A simple, fast, and accurate algorithm to estimate large phylogenies by maximum likelihood. Systematic Biology 52: 692-704.

Hewitt GM. 1999. Post-glacial re-colonization of European biota. Biological Journal of the Linnean Society 68: 87-112.

Hewitt GM. 2000. The genetic legacy of the quaternary ice ages. Nature 405: 907-913.

Hewitt GM. 2004a. Genetic consequences of climatic oscillations in the Quaternary. Philosophical Transactions of the Royal Society Series B, Biological Sciences 359: 183-195.

Hewitt GM. 2004b. The structure of biodiversity - insights from molecular phylogeography. Frontiers in Zoology 1: 4.

Hosey GR. 1982. The Bosporus land-bridge and mammal distributions in Asia Minor and the Balkans. Säugetierkunde Mitt 30: 53-62.

Hutterer RM. 2005. Order Soricomorpha. In: Wilson DE, Reeder DM, eds.. Mammal species of the world: a taxonomic and geographic reference, 3rd edn. Baltimore, MD: Johns Hopkins University Press, 220-311.

Irwin DM, Kocher TD, Wilson AC. 1991. Evolution of the cytochrome $b$ gene of Mammals. Journal of Molecular Evolution 32: 128-144.

Kimura M. 1980. A simple method for estimating evolutionary rate of base substitution through comparative studies of 
nucleotide sequences. Journal of Molecular Evolution 16: 111-120.

Michaux JR, Libois R, Paradis E, Filippucci MG. 2004. Phylogeographic history of the yellow-necked fieldmouse (Apodemus flavicollis) in Europe and in the Near and Middle East. Molecular Phylogenetics and Evolution 32: 788-798.

Nesbo CL, Fossheim T, Vollestad LA, Jakobsen KS. 1999. Genetic divergence and phylogeographic relationships among European perch (Perca fluviatilis) populations reflect glacial refugia and postglacial colonization. Molecular Ecology 8: 1387-1404.

Palme AE, Vendramin GG. 2002. Chloroplast DNA variation, postglacial recolonization and hybridization in hazel, Corylus avellana. Molecular Ecology 11: 1769-1780.

Parker SR. 1997. Sequence Navigator. Multiple sequence alignment software. Methods of Molecular Biology 70: 145154.

Posada D, Crandall KA. 1998. MODELTEST: testing the model of DNA substitution. Bioinformatics 14: 817818.

Saarma U, Ho SYW, Pybus OG, Kaljuste M, Tumanov IM, Kojola I, Vorobiev AA, Markov NI, Saveljev AP, Valdmann A, Lyapunova EA, Abramov AV, Mannil P, Korsten M, Vulla E, Pazetnov SV, Pazetnov VS, Putchkovskiy SV, Rokov AM. 2007. Mitogenetic structure of brown bears (Ursus arctos L.) in northeastern Europe and a new time frame for the formation of European brown bear lineages. Molecular Ecology 16: 401-413.

Santucci F, Emerson BC, Hewitt GM. 1998. Mitochondrial DNA phylogeography of European hedgehogs. Molecular Ecology 7: 1163-1172.

Seddon JM, Santucci F, Reeve N, Hewitt GM. 2002. Caucasus Mountains divide postulated postglacial colonization routes in the white-breasted hedgehog, Erinaceus concolor. Journal of Evolutionary Biology 15: 463-467.

Swofford DL. 1998. PAUP*. Phylogenetic Analysis Using Parsimony (* and other Methods). Version 4.0b1. Sunderland, MA: Sinauer Associates.

Taberlet P, Fumagalli L, Wust-Saucy AG, Cosson JF. 1998. Comparative phylogeography and postglacial colonization routes in Europe. Molecular Ecology 7: 453-464.

Takahata N, Slatkin M. 1984. Mitochondrial gene flow. Proceedings of the National Academy of Sciences of the United States of America, Biological Sciences 81: 17641767.

Tegelström H, Jaarola M. 1998. Geographic localization of a contact zone between bank voles Clethrionomys glareolus with distinctly different mitochondrial DNA. Acta Theriologica 43: 175-183.

Thulin CG, Fang MY, Averianov AO. 2006. Introgression from Lepus europaeus to L-timidus in Russia revealed by mitochondrial single nucleotide polymorphisms and nuclear microsatellites. Hereditas 143: 68-76. 